\title{
Classification of breast mass lesions on dynamic contrast-enhanced magnetic resonance imaging by a computer-assisted diagnosis system based on quantitative analysis
}

\author{
JIANDONG YIN $^{1}$, JIAWEN YANG ${ }^{1}$ and ZEJUN JIANG ${ }^{2}$ \\ ${ }^{1}$ Department of Radiology, Shengjing Hospital of China Medical University, Shenyang, Liaoning 110003; \\ ${ }^{2}$ Sino-Dutch Biomedical and Information Engineering School, Northeastern University, Shenyang, Liaoning 110819, P.R. China
}

Received March 8, 2018; Accepted September 28, 2018

DOI: $10.3892 /$ ol.2019.9916

\begin{abstract}
The aim of the current study was to develop a semi-automatic and quantitative method for the analysis of a time-intensity curve (TIC) from breast dynamic contrastenhanced magnetic resonance imaging. The performance of the proposed method, based on the level set segmentation algorithm, was evaluated by comparison with the traditional method. In the traditional method, the lesion area is delineated manually and the corresponding mean TIC is classified subjectively as one of three washout patterns. In addition, only one quantitative parameter, the maximum slope of increase (MSI), is calculated. In the proposed method, the lesion region was determined semi-automatically and the corresponding mean TIC was categorized quantitatively. In addition to MSI, a number of quantitative parameters were derived from the mean TIC and lesion area, including signal intensity slope $\left(\mathrm{SI}_{\text {slope }}\right)$, initial percentage of enhancement $\left(\mathrm{E}_{\text {initial }}\right)$, percentage of peak enhancement $\left(\mathrm{E}_{\text {peak }}\right)$, early signal enhancement ratio (ESER) and second enhancement percentage (SEP). Wilcoxon signed-rank test and receiver operating characteristic analyses were performed for statistical analysis. For TIC categorization the accuracy was $61.54 \%$ for the traditional method and $82.05 \%$ for the proposed method. Using the proposed method, mean curve accuracies were $84.0 \%$ for $\mathrm{SI}_{\text {slope }}, 66.7 \%$ for MSI, $66.0 \%$ for $\mathrm{E}_{\text {initial }}, 66.0 \%$ for $\mathrm{E}_{\text {peak }}, 68.0 \%$ for ESER and $44.9 \%$ for SEP. In the lesion region, the accuracies for the aforementioned parameters were 80.8, 65.4, 66.7, 62.2, 69.2 and 57.1\%, respectively. Accuracy of the MSI value derived from the traditional method was $63.4 \%$. Compared with the traditional method, the proposed semi-automatic method in the current
\end{abstract}

Correspondence to: Dr Jiandong Yin, Department of Radiology, Shengjing Hospital of China Medical University, 36 Sanhao Street, Shenyang, Liaoning 110003, P.R. China

E-mail: jiandongyin@sina.com

Key words: dynamic contrast-enhanced magnetic resonance imaging, quantitative parameter, receiver operating characteristic, semi-automatic segmentation study may provide results with a higher accuracy to differentiate benign and malignant lesions. Therefore, the proposed method should be considered as a supplementary tool for the diagnosis of breast lesions.

\section{Introduction}

Breast dynamic contrast-enhanced magnetic resonance imaging (DCE-MRI) is currently considered the most sensitive technique for the detection of breast lesions (1). In addition to morphological features, the shape of the time-intensity curve (TIC) of the signal from breast DCE-MRI has been used as an effective tool to assess possible lesion malignancy, which is highly associated with the extent of angiogenesis (2). Generally, an upslope with a quick washout pattern of the TIC is regarded as an important marker for predicting malignancy (3). Currently, in the majority of hospitals, the breast lesion is extracted manually based on the parametric map reflecting the maximum slope increase (MSI) of the TIC. The mean TIC of signals from the manually delineated area is subsequently computed, in addition to the mean MSI value. Previous studies have reported a high sensitivity for this operator-dependent method but only a low to moderate specificity for identifying benign tumors compared with malignant tumors (2,4-6).

To increase the specificity of breast malignant lesion detection, the current study devised a novel method for the analysis of TIC. Compared with the traditional method, the method proposed in the current study exhibits three different features. Firstly, the traditional method selects the representative area of the lesion manually but in the proposed method the lesion area is identified semi-automatically. Secondly, the traditional method classifies the TIC subjectively according to its shape based on three washout patterns, while the proposed method categorizes the TIC quantitatively. Finally, the traditional method only calculates one parameter, MSI, using commercial software embedded in the workstation. By contrast, several additional parameters are introduced in the current study to evaluate the performance of the proposed method in differentiating malignant lesions from benign lesions. The current study proposes that the new methodology may be useful for identifying clinical markers of breast tumor malignancy with greater specificity. This may assist the differentiation between 
malignant and benign breast lesions prior to breast conservation surgery being considered.

\section{Materials and methods}

Materials. The experimental procedures employed in the current study are presented in Fig. 1. The conventional method for TIC analysis was performed using FuncTool Performance software (version 9.4.05A; GE Healthcare, Chicago, IL, USA) embedded in a workstation. The semi-automatic diagnostic procedure was performed separately using an offline personal computer with a program developed by the current study based on MATLAB language (version R2010b; MathWorks, Natick, MA, USA).

MRI protocols and patients. The current study was approved by the Ethics Committee of Shengjing Hospital of China Medical University (Shenyang, China). All images were retrospectively selected and downloaded from the existing Picture Archiving and Communication Systems (PACS) database of the Shengjing Hospital of China Medical University (Shenyang, China), therefore the requirement for informed consent was waived. All patient information was anonymized.

DCE-MRI data were acquired using a GoldSeal Signa HDxt 3.0T scanner (GE Healthcare) with a dedicated surface multichannel receiver coil. Prior to imaging, the space between the coil and breast was filled with soft plastic foam to reduce the influence of possible patient motion on subsequent analysis. Following axial localization, dynamic examination was performed using VIBRANT-VX (GE Healthcare) with the following settings: Repetition time, $7.42 \mathrm{msec}$; echo time, $4.25 \mathrm{msec}$; flip angle, $15^{\circ}$; slice thickness, $2.2 \mathrm{~mm}$; space between slices, $2.2 \mathrm{~mm}$; inversion time, $20 \mathrm{msec}$; image matrix, 1,024x1,024; and slice number, 78. Breast DCE-MRI was performed with nine acquisitions and a temporal resolution of $80 \mathrm{sec}$ per acquisition. The first acquisition was an unenhanced baseline scan; subsequently, $0.15 \mathrm{mmol} / \mathrm{kg}$ of contrast agent (Omniscan; GE Healthcare or Magnevist; Bayer AG, Leverkusen, Germany) was administrated through an antecubital vein catheter via a power injector at $4 \mathrm{ml} / \mathrm{sec}$, followed by an equal volume of saline flush at $4 \mathrm{ml} / \mathrm{sec}$.

A total of 1,431 breast DCE-MRI images were obtained at Shengjing Hospital between January 2010 and August 2014 and were read by a radiologist with 13 years of work experience. A number of patients were referred for multiple DCE-MRI examinations prior to and following therapy, however all images collected for the current study were acquired for diagnostic purposes prior to therapeutic treatment. All of the selected lesions were single lesions presenting a mass-like shape, occurring in either the left or right breast. In addition, each lesion was confirmed as benign or malignant by biopsy or pathology (the time interval between MRI and histopathological examination was $<5$ days). Following the removal of patients that did not meet the inclusion criteria, 156 patients, with a mean age of 49.5 years (range, 23-71 years), were selected for the present study. The maximum size of the lesions ranged between 6.7 and $44.3 \mathrm{~mm}$. Details of the diagnoses verified by pathology or biopsy are presented in Table I.
Table I. Detailed histopathological diagnoses for all malignant $(n=85)$ and benign $(n=71)$ breast lesions.

\begin{tabular}{lrr}
\hline Diagnosis & $\mathrm{n}$ & $\%$ \\
\hline Malignant & 85 & 54.49 \\
Invasive ductal carcinoma & 71 & 45.51 \\
Invasive lobular carcinoma & 3 & 1.92 \\
Ductal carcinoma in situ & 4 & 2.56 \\
Phylloid tumor & 5 & 3.21 \\
Papillary carcinoma & 2 & 1.28 \\
Benign & 71 & 45.51 \\
High risk (complex sclerosing lesion, FEA, & 5 & 3.21 \\
CCC with focal atypia) & & \\
Fibroadenoma, fibroadenomatous hyperplasia & 33 & 21.15 \\
Papilloma & 4 & 2.56 \\
DH, CCC, FCC, focal fibrosis, nodular & 16 & 10.26 \\
sclerosing adenosis & & \\
Miscellaneous (chronic abscess, gynecomastia, & 13 & 8.33 \\
fat necrosis and pseudoangiomatosis) & & \\
\hline
\end{tabular}

FEA, flat epithelial atypia; CCC, columnar cell changes; DH, ductal hyperplasia; FCC, fibrocystic changes.

Traditional method: Manual extraction of lesions and qualitative analysis of TIC. Each image was downloaded from the PACS server and imported to the FuncTool Performance software by a radiologist with 22 years of experience, who was blinded to patient clinical information. A circular region of interest (ROI) was placed onto the MSI parametric map to delineate the suspicious lesion area with the most intense enhancement. The mean TIC from the ROI was observed with the naked eye and classified subjectively by its shape as one of three washout patterns: Type I (persistently enhancing), where the signal intensity continued to increase over time; type II (plateau), where the signal intensity did not change over time following its initial increase during the delayed phase; and type III (washout), where the signal intensity decreased after reaching the highest point of its initial increase during the delayed phase.

New method: Semi-automatic extraction of lesions and quantitative analysis of TIC. The current study used a series of post-processing steps to outline the suspicious lesion area.

Firstly, the third post-contrast image was subtracted from the pre-contrast image. The subtracted volume image was analyzed and the slice image with the lesion of maximum size was selected for subsequent analysis. Secondly, a rectangular ROI was drawn onto the selected slice to include the lesion, where the ROI was larger than the lesion area. This ROI image was considered as the input data. Next, a common image segmentation algorithm, the level set method (7), was applied to the input data. To improve the numerical accuracy of traditional level-set segmentation, the current study utilized a new variational formulation in which regularity was intrinsically maintained during Distance Regularized Level Set Evolution (DRLSE). Unlike the traditional method, the 


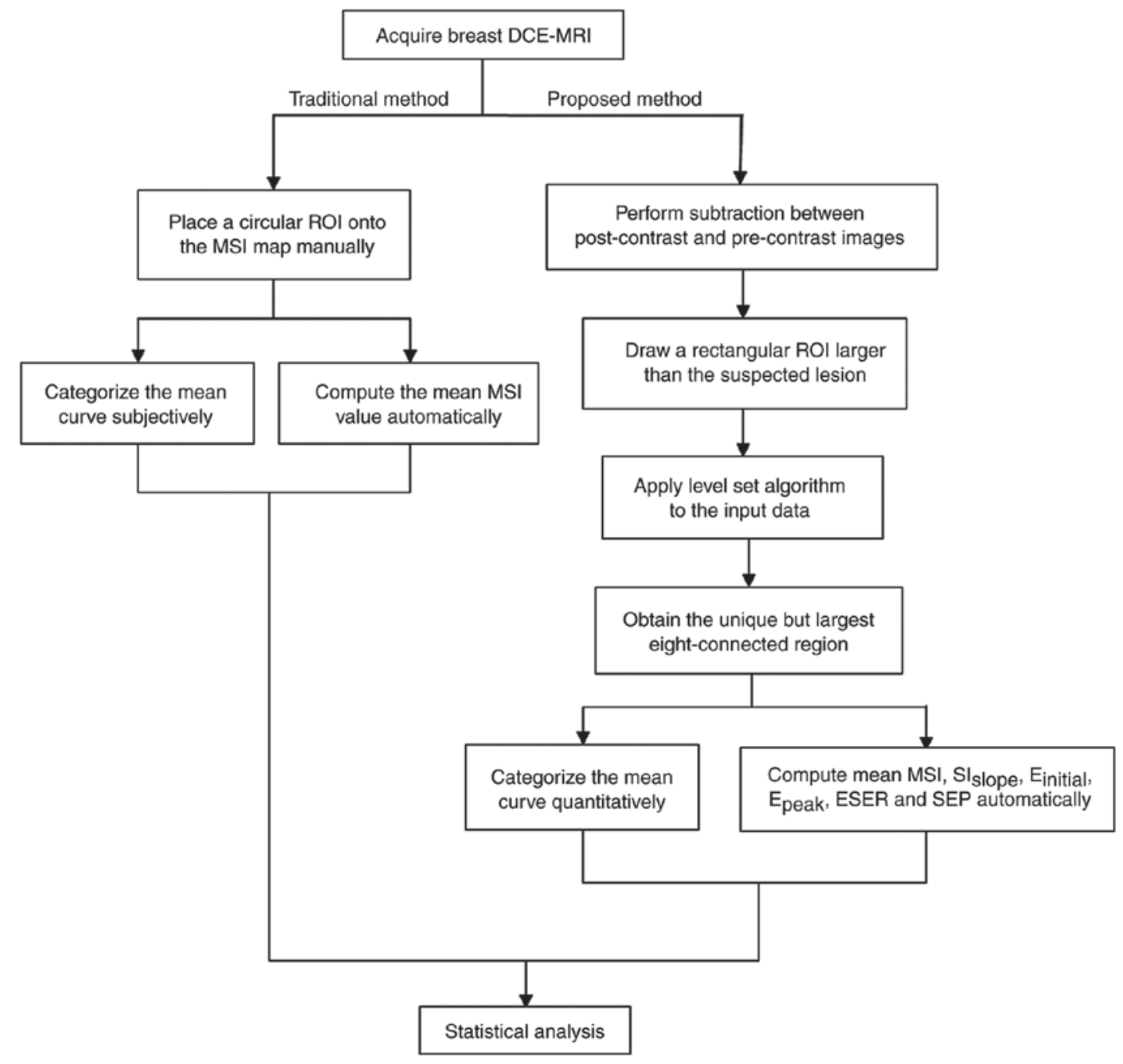

Figure 1. Flowchart outlining the experimental procedures for the traditional and proposed method of DCE-MRI analysis. ROI, region of interest; MSI, maximum slope of increase; $\mathrm{SI}_{\text {slope }}$, signal intensity slope; $\mathrm{E}_{\text {initial }}$, initial percentage of enhancement; $\mathrm{E}_{\text {peak }}$, peak enhancement percentage; ESER, early signal enhancement ratio; SEP, second enhancement percentage; DCE-MRI, dynamic contrast-enhanced magnetic resonance imaging.

DRLSE-based level set method can eliminate the need for reinitialization, which reduces numerical errors. In addition, a simpler and more efficient finite difference scheme can be adopted to conduct the DRLSE function. The effectiveness of the method used in the current study had been demonstrated by applying it to edge-based active contour models of image segmentation (7). In addition, the computational cost can be greatly reduced compared with that of the traditional level-set segmentation method. Finally, the unique and largest eightconnected region was selected as the lesion area.

Following extraction of the lesion area, quantitative analysis was performed as follows. First, the TICs from the lesion area were averaged and the mean curve was categorized quantitatively as one of three types based on the following formula: $\mathrm{SI}_{\text {slope }}=\left[\left(\mathrm{SI}_{\text {tail }}-\mathrm{SI}_{\text {mean }}\right) / \mathrm{SI}_{\text {mean }}\right] \times 100 \%$, where $\mathrm{SI}_{\text {mean }}$ is the mean value between the first two post-contrast time points and $\mathrm{SI}_{\text {tail }}$ is the signal intensity at the last time point. The mean curve was designated as type I when the $\mathrm{SI}_{\text {slope }}$ was $\geq+10 \%$, type II when the $\mathrm{SI}_{\text {slope }}$ was between $-10 \%$ and $+10 \%$ and type III when the $\mathrm{SI}_{\text {slope }}$ was $\leq-10 \%$.

The following quantitative parameters were also derived from the mean curve. $\mathrm{MSI}=\max \left(\mathrm{SI}_{\mathrm{i}+1}-\mathrm{SI}_{\mathrm{i}}\right)$, where $\mathrm{SI}_{\mathrm{i}}$ and
$\mathrm{SI}_{\mathrm{i}+1}$ denote the signal intensity of the former and latter phases, respectively, and $\mathrm{i}$ ranges between 0 and 7 . Initial percentage of enhancement $\left(\mathrm{E}_{\text {initial }}\right)=\left(\mathrm{SI}_{1}-\mathrm{SI}_{0}\right) / \mathrm{SI}_{0} \times 100$, where $\mathrm{SI}_{1}$ and $\mathrm{SI}_{0}$ represent the signal intensities of the first and pre-contrast images, respectively. The percentage of peak enhancement $\left(\mathrm{E}_{\text {peak }}\right)=\left(\mathrm{SI}_{\text {peak }}-\mathrm{SI}_{0}\right) / \mathrm{SI}_{0} \times 100$, where $\mathrm{SI}_{\text {peak }}$ represents the peak value of the contrast enhancement. The early signal enhancement ratio $(\mathrm{ESER})=\left(\mathrm{SI}_{1}-\mathrm{SI}_{0}\right) /\left(\mathrm{SI}_{2}-\mathrm{SI}_{0}\right) \times 100$, where $\mathrm{SI}_{2}$ is the intensity at the second post-contrast time point. The second enhancement percentage $(\mathrm{SEP})=\left(\mathrm{SI}_{2}-\mathrm{SI}_{0}\right) / \mathrm{SI}_{0} \times 100$.

The aforementioned parameters were also calculated for the target region on a pixel-by-pixel basis and the corresponding parametric map was color-coded. The values from the lesion area were averaged for each parameter.

Statistical methods. For TIC categorization, types II and III were designated as malignant, whereas the type I curve was designated as benign. By comparison with pathological results, the specificity, sensitivity and accuracy were calculated for the traditional subjective method and the new proposed quantitative method. A paired-sample Wilcoxon's test was performed using SPSS software (version 16.0; SPSS, Inc., Chicago, IL, 

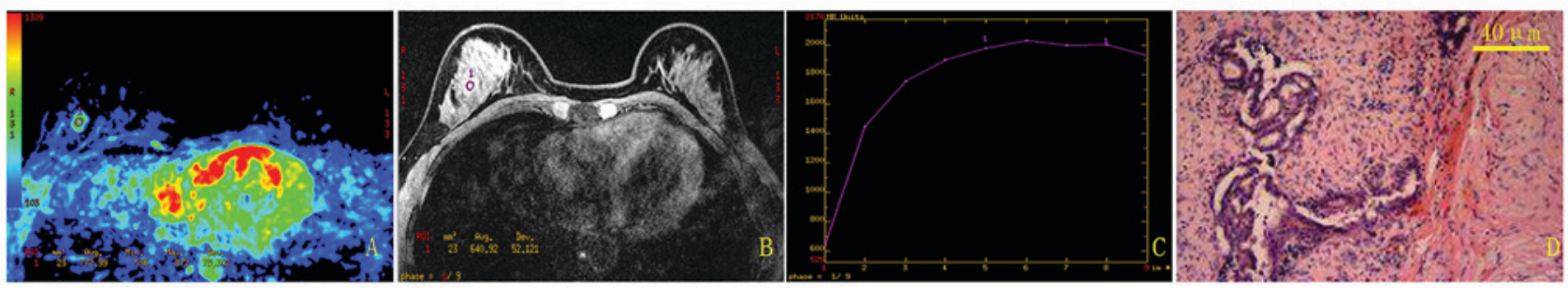

Figure 2. Results from the traditional method for a randomly selected case. (A) MSI map. (B) Pre-contrast image. (C) Mean curve of the time-intensity curves from the manually drawn region of interest. (D) Pathological result demonstrated breast adenosis combined with focal fibroadenomatoid hyperplasia. The mean MSI value was 772.99. MSI, maximum slope of increase.

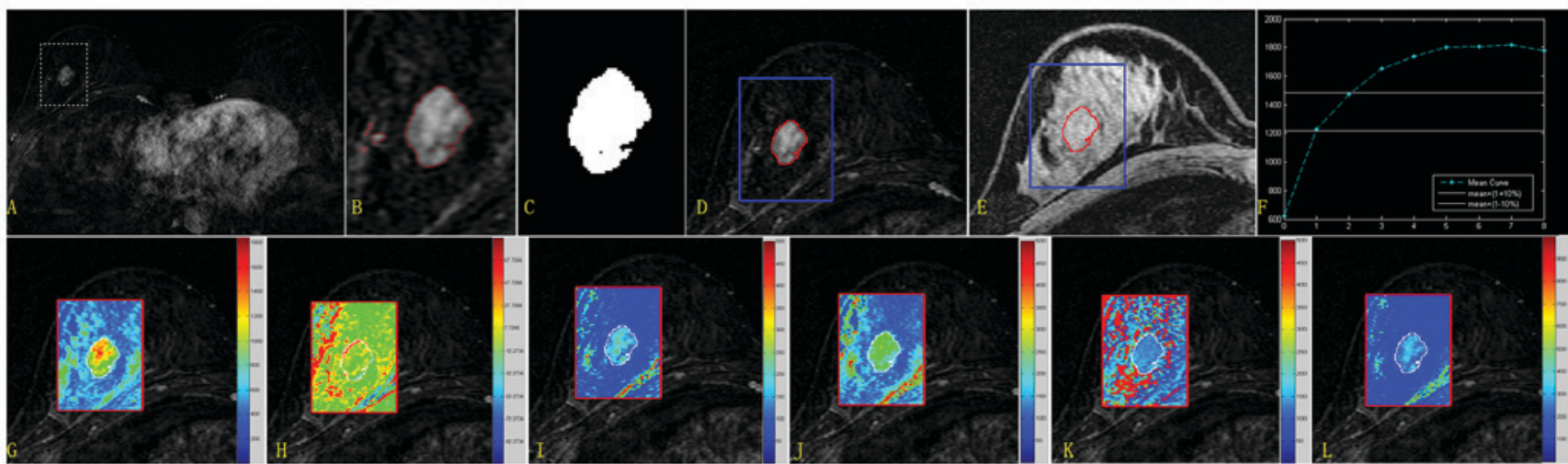

Figure 3. Extraction procedure for the lesion area and quantitative results from the new method for the randomly selected case. Upper row: Extraction of the lesion region and quantitative categorization of the mean TIC. (A) Subtracted image for subsequent analysis covered by a rectangular ROI. (B) Segmented image based on the level set algorithm (only the ROI is presented). (C) The unique but largest eight-connected image was considered the target region. (D) Enlarged subtracted image covered by the lesion margin (red, lesion borderline; blue, ROI borderline). (E) The lesion margin superimposed on the pre-contrast image. (F) Mean TIC. Mean values calculated for the parameters $\mathrm{SI}_{\text {slope }}, \mathrm{E}_{\text {initial }}, \mathrm{E}_{\text {peak }}$, ESER, MSI and SEP were 31.979, 100.421, 197.066, 71.683, 622.287 and 140.089, respectively. Lower row: Quantitative parametric maps for the ROI image. (G) MSI map. (H) SI $I_{\text {slope }}$ map. (I) $E_{\text {initial }}$ map. (J) $E_{\text {peak }}$ map. (K) ESER map. (L) SEP map. Mean values calculated for $\mathrm{SI}_{\text {slope }}, \mathrm{E}_{\text {initial }}, \mathrm{E}_{\text {peak }}$, ESER, MSI and SEP were 34.237, 101.577, 207.811, 71.4369, 1276.007 and 141.387, respectively. TIC, time-intensity curve; ROI, region of interest; $\mathrm{SI}_{\text {slope }}$, signal intensity slope; $\mathrm{E}_{\text {initial }}$, initial percentage of enhancement; $\mathrm{E}_{\text {peak }}$, percentage of peak enhancement; ESER, early signal enhancement ratio; MSI, maximum slope of increase; SEP, second enhancement percentage.

USA) to compare the two methods. $\mathrm{P}<0.05$ was considered to indicate a statistically significant difference.

For each quantitative parameter, a receiver operating characteristic (ROC) curve was generated using the statistical software MedCalc (version 14.10.20; http://www.medcalc.org/) and the area under the ROC curve (AUC) was used as an index of diagnostic performance. The optimal cutoff value was also obtained using MedCalc for each parameter, from which the specificity, sensitivity and accuracy were determined.

\section{Results}

The current study used a randomly selected patient (benign; 39 years old) to illustrate the results of the traditional method (Fig. 2) and the new method (Fig. 3). Using the traditional method, the mean TIC for this patient was categorized as type II, which was a false positive result. In comparison, using the new semi-automatic quantitative method, the mean TIC was classified as type I, which agreed with the pathological result.

For the two methods, the statistical results of TIC categorization are presented in Table II. A significant difference was identified in the diagnostic accuracy between the traditional method and the quantitative method proposed in the current study $(\mathrm{Z}=-6.594, \mathrm{P}<0.001)$. For the quantitative parameters,
Table II. Time intensity curve categorization results obtained using the traditional method and proposed semi-automatic method.

\begin{tabular}{lcc}
\hline Parameter & Traditional method & Proposed method \\
\hline Sensitivity, \% & 85.9 & 83.5 \\
Specificity, \% & 32.4 & 80.3 \\
Accuracy, \% & 61.5 & 82.1 \\
\hline
\end{tabular}

the statistical results are presented in Fig. 4 and Table III. The highest accuracy $(84.0 \%)$ was obtained based on the parameter $\mathrm{SI}_{\text {slope }}$, derived from the mean TIC. Compared with the traditional method, the new method demonstrated a higher sensitivity (84.7\% vs. $64.7 \%)$ and specificity (83.1\% vs. $62.0 \%)$.

\section{Discussion}

Breast carcinoma is the most common cancer type and a leading cause of mortality in females worldwide (8). Traditional examination techniques, including X-ray, mammography and ultrasonography are routinely utilized in clinical practice to predict tumor chemo-responsiveness. 

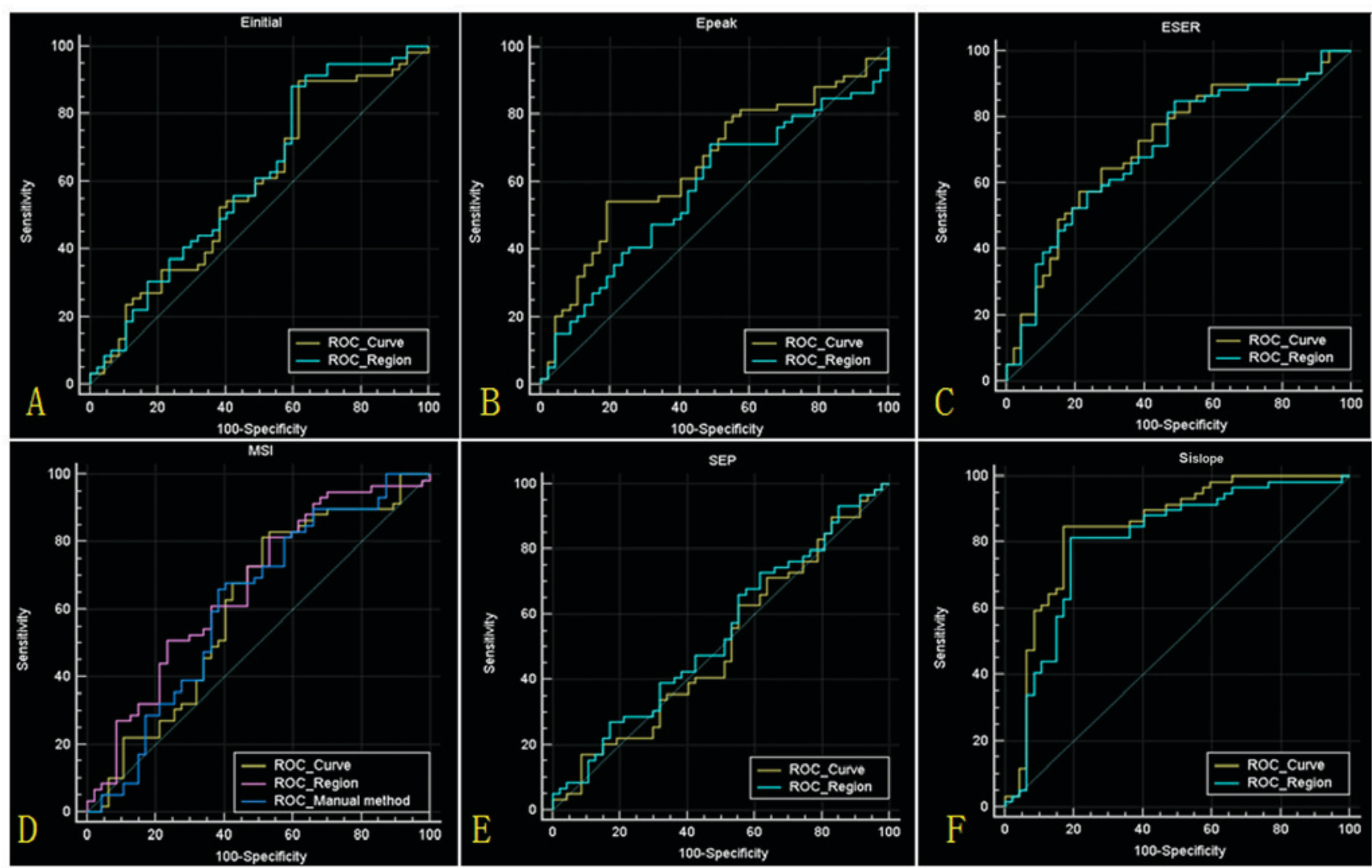

Figure 4. Results of ROC analyses. (A-F) ROC curves for $\mathrm{E}_{\text {initial }}, \mathrm{E}_{\text {peak }}$, ESER, MSI, SEP and $\mathrm{SI}_{\text {slope }}$ values, respectively, calculated from the mean time intensity curve (ROC_Curve) and lesion region (ROC_Region). (D) The ROC curve for MSI values calculated from the traditional method (ROC_Manual method) is also indicated. ROC, receiver operating characteristic; $\mathrm{E}_{\text {initial }}$, initial percentage of enhancement; $\mathrm{E}_{\text {peak }}$, percentage of peak enhancement; ESER, early signal enhancement ratio; MSI, maximum slope of increase; SEP, second enhancement percentage; $\mathrm{SI}_{\text {slope }}$, signal intensity slope.

However, these techniques exhibit only low to moderate associations with histologically verified pathology following breast surgery $(4,7,9,10)$. DCE-MRI with repetitive measurements following contrast media administration is a frequently used technique in the diagnosis and staging of breast cancer lesions $(5,6,11,12)$. Furthermore, DCE-MRI has the ability to reliably monitor the effect of neoadjuvant chemotherapy in the treatment of breast cancer $(4,13,14)$. TICs of signals from DCE-MRI differ between malignant and benign breast lesions are strongly associated with tumor angiogenesis and provide insights into lesion pathophysiology $(15,16)$. For malignant cases, a TIC curve typically exhibits a strong initial enhancement of signal followed by a plateau or washout; by contrast, benign lesions typically present continuously increasing time courses. However, overlap exists between the enhancement curve types of benign and malignant lesions. For traditional interpretation of DCE-MRI, the manual placement of the ROI to delineate a contrast-enhancing lesion is subjective and may lead to inaccuracies if the ROI is placed in a less vital area of the lesion. In addition, categorization of TIC according to its shape is subjective, which may result in low diagnostic accuracy (17). The reported specificity for the traditional method in distinguishing benign from malignant lesions is relatively low and high false-positive rates may result in high biopsy rates, which not only unnecessarily increase health care costs, but may also harm numerous cancer-free females with long-term psychological consequences $(18,19)$.
To obtain higher diagnostic specificity, the current study has proposed a novel method for TIC analysis, which determines the lesion area semi-automatically, objectively categorizes the TIC as one of the three washout patterns and provides a higher number of quantitative parameters reflecting the enhancement information. The results identified that the proposed method demonstrated improved performance in distinguishing benign from malignant lesions compared with the traditional method. For TIC classification, although the sensitivity of the traditional method was high, its specificity was low, which is in agreement with a number of previous studies (20-25). Low specificity in DCE-MRI diagnosis may lead to low accuracy. Compared with the traditional method, the new method slightly decreased the sensitivity, but substantially increased the specificity when using the parameter $\mathrm{SI}_{\text {slope }}$ derived from the mean curve. Therefore, overall accuracy was improved. Traditionally, the optimal threshold value is set at $+10 \%$ (14). However, the current study identified that diagnostic accuracy was improved if the value was set at $+9.30 \%$. The current study proposes that the establishment of a new optimal cutoff value may improve future interpretations of breast DCE-MRIs, eliminating unnecessary surgeries and biopsies for benign lesions. The results presented in the current study may be useful for guiding future studies and may lead to further retrospective analyses of similar datasets.

Previously, a number of studies investigating computeraided diagnosis of breast DCE-MRI have been performed with the aim of improving the differentiation of benign from 
Table III. Mean curve statistical analysis for the quantitative parameters.

A, Proposed method

\begin{tabular}{|c|c|c|c|c|c|c|c|}
\hline Parameter & AUC & SE & $95 \% \mathrm{CI}$ & Optimal cutoff & Sensitivity, \% & Specificity, \% & Accuracy, $\%$ \\
\hline \multicolumn{8}{|c|}{$\begin{array}{l}\text { Mean time } \\
\text { intensity curve }\end{array}$} \\
\hline $\mathrm{Si}_{\text {slope }}$ & 0.846 & 0.0409 & $0.766,0.927$ & $\leq 9.296$ & 84.7 & 83.1 & 84.0 \\
\hline MSI & 0.611 & 0.0573 & $0.498,0.723$ & $>682.142$ & 81.2 & 49.3 & 66.7 \\
\hline $\mathrm{E}_{\text {initial }}$ & 0.590 & 0.0568 & $0.479,0.701$ & $>112.81$ & 89.4 & 38.0 & 66.0 \\
\hline $\mathrm{E}_{\text {peak }}$ & 0.652 & 0.0538 & $0.547,0.758$ & $\leq 210.934$ & 54.1 & 80.3 & 66.0 \\
\hline ESER & 0.716 & 0.0507 & $0.617,0.816$ & $>77.146$ & 64.7 & 72.8 & 68.0 \\
\hline SEP & 0.505 & 0.0574 & $0.392,0.617$ & $>205.264$ & 41.2 & 49.3 & 44.9 \\
\hline \multicolumn{8}{|c|}{ Lesion region } \\
\hline $\mathrm{Si}_{\text {slope }}$ & 0.802 & 0.0461 & $0.712,0.892$ & $\leq 16.952$ & 81.2 & 80.3 & 80.8 \\
\hline MSI & 0.663 & 0.0539 & $0.558,0.769$ & $\leq 1503.171$ & 81.2 & 46.5 & 65.4 \\
\hline $\mathrm{E}_{\text {initial }}$ & 0.611 & 0.0565 & $0.500,0.722$ & $>115.984$ & 88.2 & 40.9 & 66.7 \\
\hline$E_{\text {peak }}$ & 0.576 & 0.0561 & $0.466,0.686$ & $\leq 308.249$ & 71.8 & 50.7 & 62.2 \\
\hline ESER & 0.705 & 0.0514 & $0.604,0.806$ & $>70.063$ & 84.7 & 50.7 & 69.2 \\
\hline SEP & 0.537 & 0.0570 & $0.426,0.649$ & $>180.913$ & 73.0 & 38.0 & 57.1 \\
\hline
\end{tabular}

B, Traditional method

\begin{tabular}{lccccccc}
\hline Parameter & AUC & SE & $95 \%$ CI & Optimal cutoff & Sensitivity, \% & Specificity, \% & Accuracy, \% \\
\hline MSI & 0.604 & 0.0572 & $0.503,0.699$ & $>884.427$ & 64.7 & 62.0 & 63.4 \\
\hline
\end{tabular}

AUC, area under the curve; $\mathrm{CI}$, confidence interval; $\mathrm{SI}_{\text {slope }}$, signal intensity slope; MSI, maximum slope of increase; $\mathrm{E}_{\text {initial }}$, initial percentage of enhancement; $E_{\text {peak }}$, percentage of peak enhancement; ESER, early signal enhancement ratio; SEP, second enhancement percentage; SE, standard error.

malignant lesions $(6,15,16,19,21,26-29)$. A computerized detection scheme to compute a global contrast-enhanced feature was proposed by Yang et al (19), which achieved a diagnostic sensitivity of $91.3 \%$, but a specificity of only $66 \%$. A semi-automatic lesion segmentation system based on a supervised learning formulation was reported by Levman et al (26). In contrast to the traditional enhancement threshold method, AUC indexing improved diagnostic performance from 0.75 to 0.79 . In studies by El Khouli et al (21) and Newell et al (29), parameters reflecting hemodynamic information were measured to distinguish between benign and malignant lesions, resulting in higher performance compared with conventional kinetic curve analysis. In an innovative study (16), kinetic curve and morphological features were analyzed quantitatively and a morphodynamic index (MDI) was presented. With an MDI cutoff value of 50\%, the sensitivity and specificity were reported as 96.5 and $75.5 \%$, respectively. Compared with these studies, the method proposed in the current study provides a higher number of quantitative parameters reflecting the enhancement information of breast lesions. The maximum AUC (0.846) in the current study, with sensitivity of 84.7 and specificity of $83.1 \%$, was obtained from $\mathrm{SI}_{\text {slope }}$ values measured from the mean curve of the semiautomatically extracted lesion, with a corresponding accuracy of $84.0 \%$. The diagnostic accuracy may be even higher if morphological features were also analyzed. In real clinical practice, breast MRI findings should also be interpreted based on the following three characteristics according to the American College of Radiology Breast Imaging Reporting and Data System MRI criteria: i) Shape (round, oval, lobular or irregular), ii) margin (smooth, irregular or spiculated) and iii) internal enhancement (homogeneous, heterogeneous, rim enhancement, dark internal septa, enhancing internal septa, central enhancement or no enhancement) (30). In addition, other specific findings identified by MRI, including apparent diffusion coefficient values, should be analyzed to increase diagnostic accuracy.

Two limitations of the current study should be emphasized. First, the sample size in the current study was insufficient to obtain a definitive conclusion. If the sample size was changed, the optimal cutoff value and accuracy rate may change accordingly. Second, the current study only analyzed TICs from breast DCE-MRI and did not utilize morphological features of the lesions for tumor diagnosis, which may have further improved the diagnostic accuracy of breast DCE-MRI using the proposed semi-automatic method $(2,31,32)$.

In summary, experimental results suggest that the proposed method in the current study may improve the accuracy of DCE-MRI in distinguishing benign from malignant breast 
lesions. Furthermore, the proposed method may be a useful supplementary tool to assist with the subjective interpretation of a DCE-MRI made by a radiologist.

\section{Acknowledgements}

Not applicable.

\section{Funding}

The present study was financially supported by the Science and Technique Foundation of Liaoning (grant no. 2011402016) and the Startup Foundation for Doctors of Liaoning Province (grant no. 201601118).

\section{Availability of data and materials}

The datasets used and/or analyzed during the present study are available from the corresponding author on reasonable request.

\section{Authors' contributions}

JDY was responsible for the experimental design. JWY collected the general data of patients. JWY and ZJ conducted the quantitative analysis. All authors read and approved the final manuscript.

\section{Ethics approval and consent to participate}

The present study was approved by the Ethics Committee of Shengjing Hospital of China Medical University (Shenyang, China). As this was a retrospective study, the requirement for informed consent was waived. All patient information was anonymized.

\section{Patient consent for publication}

Not applicable.

\section{Competing interests}

The authors declare that they have no competing interests.

\section{References}

1. Kuhl CK, Schrading S, Bieling HB, Wardelmann E, Leutner CC, Koenig R, Kuhn W and Schild HH: MRI for diagnosis of pure ductal carcinoma in situ: A prospective observational study. Lancet 370: 485-492, 2007.

2. Chang YC, Huang YH, Huang CS, Chang PK, Chen JH and Chang RF: Classification of breast mass lesions using modelbased analysis of the characteristic kinetic curve derived from fuzzy c-means clustering. Magn Reson Imaging 30: 312-322, 2012.

3. Vag T, Baltzer PA, Dietzel M, Zoubi R, Gajda M, Camara O and Kaiser WA: Kinetic analysis of lesions without mass effect on breast MRI using manual and computer-assisted methods. Eur Radiol 21: 893-898, 2011.

4. Böttcher J, Renz DM, Zahm DM, Pfeil A, Fallenberg EM, Streitparth F, Maurer MH, Hamm B and Engelken FJ: Response to neoadjuvant treatment of invasive ductal breast carcinomas including outcome evaluation: MRI analysis by an automatic CAD system in comparison to visual evaluation. Acta Oncol 53 759-768, 2014.
5. Renz DM, Böttcher J, Baltzer PA, Dietzel M, Vag T, Gajda M, Camara O, Runnebaum IB and Kaiser WA: The contralateral synchronous breast carcinoma: A comparison of histology, localization, and magnetic resonance imaging characteristics with the primary index cancer. Breast Cancer Res Treat 120: 449-459, 2010.

6. Renz DM, Diekmann F, Schmitzberger FF, Pietsch H, Fallenberg EM, Durmus T, Huppertz A, Böttcher J, Bick U, Hamm B, et al: Pharmacokinetic approach for dynamic breast MRI to indicate signal intensity time curves of benign and malignant lesions by using the tumor flow residence time. Invest Radiol 48: 69-78, 2013.

7. Li C, Xu C, Gui C and Fox MD: Distance regularized level set evolution and its application to image segmentation. IEEE Trans Image Process 19: 3243-3254, 2010.

8. Sun L, Chen G, Zhou Y, Zhang L, Jin Z, Liu W, Wu G, Jin F Li K and Chen B: Clinical significance of MSKCC nomogram on guiding the application of touch imprint cytology and frozen section in intraoperative assessment of breast sentinel lymph nodes. Oncotarget 8: 78105-78112, 2017

9. Croshaw R, Shapiro-Wright H, Svensson E, Erb K and Julian T: Accuracy of clinical examination, digital mammogram, ultrasound, and MRI in determining postneoadjuvant pathologic tumor response in operable breast cancer patients. Ann Surg Oncol 18: 3160-3163, 2011.

10. Sardanelli F, Podo F, Santoro F, Manoukian S, Bergonzi S, Trecate G, Vergnaghi D, Federico M, Cortesi L, Corcione S, et al; High Breast Cancer Risk Italian 1 (HIBCRIT-1) Study: Multicenter surveillance of women at high genetic breast cancer risk using mammography, ultrasonography, and contrastenhanced magnetic resonance imaging (the high breast cancer risk italian 1 study): Final results. Invest Radiol 46: 94-105, 2011.

11. Lehman CD, Gatsonis C, Kuhl CK, Hendrick RE, Pisano ED, Hanna L, Peacock S, Smazal SF, Maki DD, Julian TB, et al; ACRIN Trial 6667 Investigators Group: MRI evaluation of the contralateral breast in women with recently diagnosed breast cancer. N Engl J Med 356: 1295-1303, 2007.

12. Pediconi F, Miglio E, Telesca M, Luciani ML, Kirchin MA, Passariello $\mathrm{R}$ and Catalano $\mathrm{C}$ : Effect of preoperative breast magnetic resonance imaging on surgical decision making and cancer recurrence rates. Invest Radiol 47: 128-135, 2012.

13. Johansen R, Jensen LR, Rydland J, Goa PE, Kvistad KA, Bathen TF, Axelson DE, Lundgren $S$ and Gribbestad IS: Predicting survival and early clinical response to primary chemotherapy for patients with locally advanced breast cancer using DCE-MRI. J Magn Reson Imaging 29: 1300-1307, 2009.

14. Abramson RG, Li X, Hoyt TL, Su PF, Arlinghaus LR, Wilson KJ, Abramson VG, Chakravarthy AB and Yankeelov TE: Early assessment of breast cancer response to neoadjuvant chemotherapy by semi-quantitative analysis of high-temporal resolution DCE-MRI: Preliminary results. Magn Reson Imaging 31: 1457-1464, 2013

15. Baltzer PA, Renz DM, Kullnig PE, Gajda M, Camara O and Kaiser WA: Application of computer-aided diagnosis (CAD) in MR-mammography (MRM): Do we really need whole lesion time curve distribution analysis? Acad Radiol 16: 435-442, 2009.

16. Renz DM, Böttcher J, Diekmann F, Poellinger A, Maurer MH, Pfeil A, Streitparth F, Collettini F, Bick U, Hamm B, et al: Detection and classification of contrast-enhancing masses by a fully automatic computer-assisted diagnosis system for breast MRI. J Magn Reson Imaging 35: 1077-1088, 2012.

17. Beresford MJ, Padhani AR, Taylor NJ, Ah-See ML, Stirling JJ, Makris A, d'Arcy JA and Collins DJ: Inter- and intraobserver variability in the evaluation of dynamic breast cancer MRI. J Magn Reson Imaging 24: 1316-1325, 2006.

18. Brodersen J and Siersma VD: Long-term psychosocial consequences of false-positive screening mammography. Ann Fam Med 11: 106-115, 2013.

19. Yang Q, Li L, Zhang J, Shao G and Zheng B: A computerized global MR image feature analysis scheme to assist diagnosis of breast cancer: A preliminary assessment. Eur J Radiol 83: 1086-1091, 2014.

20. Partridge SC, Rahbar H, Murthy R, Chai X, Kurland BF, DeMartini WB and Lehman CD: Improved diagnostic accuracy of breast MRI through combined apparent diffusion coefficients and dynamic contrast-enhanced kinetics. Magn Reson Med 65: 1759-1767, 2011.

21. El Khouli RH, Macura KJ, Kamel IR, Jacobs MA and Bluemke DA: 3-T dynamic contrast-enhanced MRI of the breast: Pharmacokinetic parameters versus conventional kinetic curve analysis. AJR Am J Roentgenol 197: 1498-1505, 2011. 
22. Bluemke DA, Gatsonis CA, Chen MH, DeAngelis GA, DeBruhl N, Harms S, Heywang-Köbrunner SH, Hylton N, Kuhl CK, Lehman C, et al: Magnetic resonance imaging of the breast prior to biopsy. JAMA 292: 2735-2742, 2004.

23. Choi HK, Cho N, Moon WK, Im SA, Han W and Noh DY: Magnetic resonance imaging evaluation of residual ductal carcinoma in situ following preoperative chemotherapy in breast cancer patients. Eur J Radiol 81: 737-743, 2012

24. Pinker-Domenig K, Bogner W, Gruber S, Bickel H, Duffy S, Schernthaner M, Dubsky P, Pluschnig U, Rudas M, Trattnig S, et al: High resolution MRI of the breast at 3 T: Which BI-RADS ${ }^{\circledR}$ descriptors are most strongly associated with the diagnosis of breast cancer? Eur Radiol 22: 322-330, 2012.

25. Pinker K, Bogner W, Baltzer P, Gruber S, Bickel H, Brueck B, Trattnig S, Weber M, Dubsky P, Bago-Horvath Z, et al: Improved diagnostic accuracy with multiparametric magnetic resonance imaging of the breast using dynamic contrast-enhanced magnetic resonance imaging, diffusion-weighted imaging, and 3-dimensional proton magnetic resonance spectroscopic imaging. Invest Radiol 49: 421-430, 2014.

26. Levman J, Warner E, Causer P and Martel A: Semi-automatic region-of-interest segmentation based computer-aided diagnosis of mass lesions from dynamic contrast-enhanced magnetic resonance imaging based breast cancer screening. J Digit Imaging 27: 670-678, 2014.

27. Williams TC, DeMartini WB, Partridge SC, Peacock S and Lehman CD: Breast MR imaging: Computer-aided evaluation program for discriminating benign from malignant lesions. Radiology 244: 94-103, 2007.
28. Renz DM, Durmus T, Böttcher J, Taupitz M, Diekmann F, Huppertz A, Pfeil A, Maurer MH, Streitparth F, Bick U, et al: Comparison of gadoteric acid and gadobutrol for detection as well as morphologic and dynamic characterization of lesions on breast dynamic contrast-enhanced magnetic resonance imaging. Invest Radiol 49: 474-484, 2014.

29. Newell D, Nie K, Chen JH, Hsu CC, Yu HJ, Nalcioglu O and Su MY: Selection of diagnostic features on breast MRI to differentiate between malignant and benign lesions using computer-aided diagnosis: Differences in lesions presenting as mass and non-mass-like enhancement. Eur Radiol 20: 771-781, 2010.

30. D'Orsi CJ, Sickles EA, Mendelson EB, Morris EA (eds.): ACR BI-RADS ${ }^{\circledR}$ Atlas, Breast Imaging Reporting and Data System. American College of Radiology, Reston, VA, 2013.

31. Kong Y, Deng Y and Dai Q: Discriminative clustering and feature selection for brain MRI segmentation. IEEE Signal Process Lett 22: 573-577, 2015.

32. Kong Y, Li Y, Wu J and Shu H: Noise reduction of diffusion tensor images by sparse representation and dictionary learning. Biomed Eng Online 15: 5, 2016.

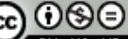

This work is licensed under a Creative Common Attribution-NonCommercial-NoDerivatives 4.0 International (CC BY-NC-ND 4.0) License. 